\title{
No sólo la apertura define a los estudiantes con mayor potencial creativo
}

\author{
María José Ruiz-Melero ${ }^{1}$, Rosario Bermejo ${ }^{1}$, \\ Mercedes Ferrando ${ }^{1}$ y Marta Sainz ${ }^{1}$
}

${ }^{1}$ Departamento de Psicología Evolutiva y de la Educación, Universidad de Murcia, Murcia

\section{España}

Correspondencia: María José Ruiz Melero. Universidad de Murcia, Campus de Espinardo. 30100 (Murcia). España. E-mail: mariajose.ruiz4@um.es

(C) Universidad de Almería and Ilustre Colegio Oficial de la Psicología de Andalucía Oriental (Spain) 


\section{Resumen}

Introducción. La relevancia de estudiar los rasgos de personalidad en los estudiantes creativos dentro del ámbito educativo implica su identificación y posterior respuesta educativa adecuada a los diferentes perfiles creativos que manifiestan. Por tanto, el objetivo fue analizar la relación entre los rasgos de la personalidad y las habilidades creativas, así como profundizar en qué rasgos de personalidad son los que definen a los estudiantes con mayor potencial creativo en una muestra de adolescentes, basándonos en el modelo de los Cinco Grandes Factores de la personalidad (Costa \& McCrae, 1992).

Método. Participaron 178 estudiantes de Educación Secundaria Obligatoria (ESO) de la Región de Murcia (España), con edades entre los 12 y los 17 años $(M=14.59 ; D T=1.40)$. De los cuales un $49.3 \%$ eran varones. Los instrumentos utilizados fueron: el TTCT (Torrance, 1974), para valorar la creatividad; el BFQ-NA (Big Five Questionnaire de Personalidad para niños y adolescentes; Barbaranelli, Caprara y Rabasca, 1998), en concreto, la adaptación española (Del Barrio, Carrasco \& Holgado, 2006) y el NEO-FFI (Inventario de Personalidad NEO Revisado, versión reducida; Costa \& McCrae, 2008), para los rasgos de personalidad. Para valorar las diferencias en personalidad realizamos tres grupos en función de su nivel de creatividad (alta, media y baja).

Resultados.-Los datos sugieren que fueron tres los rasgos de la personalidad relacionados con las dimensiones creativas: la Extraversión, la Conciencia/Responsabilidad y la Apertura. Mientras que, las personas más creativas presentaron puntuaciones significativamente más elevadas en los siguientes rasgos de personalidad: Extraversión, Conciencia/Responsabilidad y Amabilidad.

Discusión y conclusiones. A nivel general se observa cierta relación entre las habilidades creativas y los rasgos de personalidad. Concretante son la Extraversión, la Conciencia/Responsabilidad y la Apertura las dimensiones más relacionadas con la creatividad. De las cuales, la Extraversión y la Conciencia/Responsabilidad son las que definen en mayor medida un perfil creativo. Resultados en esta línea son los obtenidos por Chamorro-Premuzic y Reichenbacher (2008), Furnham (2015), y Szobiová (2006).

Palabras Clave: creatividad, personalidad, extraversión, apertura. 


\section{Abstract}

Introduction: The relevance to study personality traits in creative students within the educational field implies their identification and then select the right educational response to the different creative profiles of the students. The aim was to analyze the relationship between personality and creativity, and to deepen into which personality traits defines the students with the greatest creative potential in a sample of adolescents, based on the model of the Five Factors Model of personality (Costa \& McCrae, 1992).

Method: Participants were 178 students of Compulsory Secondary Education (ESO) from the Region of Murcia (Spain), aged between 12 and 17 years old $(M=14.59, S D=1.40)$. Of which $49.3 \%$ were boys. The instruments used in our study have been: to value creativity, the TTCT (Torrance, 1974); and to evaluate personality traits, the BFQ-NA (Big Five Personality Questionnaire for children and teenagers, Barbaranelli, Caprara \& Rabasca, 1998), specifically, the adaptation to Spanish (Del Barrio, Carrasco \& Holgado, 2006) or the NEO-FFI (Revised NEO Personality Inventory, reduced version, Costa \& McCrae, 2008), according to the age of the participants.

Results: To assess the differences in personality, we made three groups based on their level of creativity (high, medium and low). The results showed that there were three personality traits were related to creative dimensions: Extraversion, Consciousness and Openness. While, the most creative people present significantly higher scores on the following personality traits: Extraversion, Consciousness and Agreeableness.

Discussion or Conclusion: In general, there was a moderate relationship between creative skills and personality traits. Extraversion, Consciousness and Openness were the personality traits the most related to creativity. Of which, Extraversion and Consciousness were the most define a creative profile. Similar results were those obtained by Chamorro-Premuzic \& Reichenbacher (2008), Furnham (2015), and Szobiová (2006).

Keywords: creativity, personality, extraversion, openness. 


\section{Introducción}

El estudio de la relación entre la personalidad y la creatividad es una constante en la literatura científica. La relación entre ambos es muy compleja e influyen diferentes condicionantes tanto biológicos como personales y contextuales (Feist, 2010; 2019). En este trabajo hemos analizado los rasgos de personalidad asociados a la creatividad y al logro creativo, así como, qué rasgos diferencian a las personas con mayor potencial creativo de las que lo presentan en menor medida.

De acuerdo con Selby, Shaw y Houtz (2005) el estudio de la personalidad creativa se ha planteado como una importante línea de investigación en relación con la creatividad. Algunos autores tratan de ahondar en aquellas características personales que definen a las personas más creativas (Feist y Barron, 2003; Szobiová, 2006). Y otros autores, como McCrae y Greenberg (2014) se preguntan si son determinados rasgos de personalidad los que llevan a los genios creativos a afrontar los problemas, tareas o situaciones de una forma diferente. Según los autores, a los genios creativos se les atribuyen ciertos dones que los llevan a resolver problemas artísticos, intelectuales y prácticos de formas originales. Indicando que es la Apertura a la experiencia la característica clave de estos. Según estos autores, independientemente del campo de conocimiento en el que trabajen, una de las características distintivas de estos genios son sus contribuciones excepcionales y la atracción que sienten para realizar nuevas combinaciones de ideas.

Simonton (2000) indicó que la ambición era una característica definitoria de estas personas altamente creativas, y que estaba asociada con la dimensión de Conciencia/Responsabilidad. Diferentes estudios han analizado la personalidad de figuras que han sido consideradas como creativas en su ámbito de trabajo (Skinner y Ford), personas que representaban dos polos opuestos de personalidad y creatividad. El estudio lo hicieron a través del análisis de documentos y testimonios de personas que los conocieron. En el caso de Skinner, gran figura creativa dentro del campo del conductismo, indicaron que tenía un perfil caracterizado por una alta Conciencia/Responsabilidad y Apertura a la experiencia, un cierto grado de Neuroticismo y Extraversión, y no fue considerado ni amable ni desagradable (Overskeid, Grønnerød y Simonton, 2012). En cambio, en el caso de Henri Ford, quien revolucionó el campo de la ingeniería mecánica, su perfil responde a una persona con alta Conciencia/Responsabilidad, sin embargo, parece que se resistía a cambiar su primer modelo de coche, a pesar de las demandas del 
contexto. Ford tuvo una gran motivación intrínseca sólo por aquellas cosas que le interesaban, lo cual ha hecho que se le etiquete como una persona poco abierta a las nuevas experiencias no contempladas dentro de sus intereses (McCrae y Greenberg, 2014). Los resultados de estas investigaciones pueden indicar que los rasgos de personalidad difieren en función del dominio donde se manifieste el potencial creativo (Bernal, Esparza, Ruiz, Ferrando y Sainz, 2017).

Las características personales influyen de manera natural en la manifestación creativa. Pero ¿cuáles son las cualidades que aumentan la probabilidad de aparición de ideas creativas en unas personas respecto a otras? ¿Son los estilos cognitivos, las actitudes, la historia de desarrollo, los estilos motivacionales o los rasgos de la personalidad? (Feist, 2018). En este sentido, se afirma que la creatividad es el resultado de múltiples factores como son: los procesos cognitivos, los afectivos, los comportamentales y los contextuales (Amabile, Barsade, Mueller, y Staw, 2005; da Costa, Páez, Sánchez, Garaigordobil y Gondim, 2015; Sternberg y Lubart, 1995/1997).

Entre los factores personales cabe destacar: los rasgos de personalidad, la inteligencia, el conocimiento, el estilo de pensamiento y la motivación (Sternberg y Lubart, 1995/1997). Respecto a los rasgos de personalidad, aunque ninguno de ellos pueda garantizar la producción creativa, parece haber cierto acuerdo sobre la existencia de determinados rasgos en los individuos que más han desarrollado su creatividad (Romo-Santos, Sánchez-Ruiz y AlfonsoBenlliure, 2017).

En este sentido, en el estudio de la personalidad creativa desde el modelo de Eysenck y Eysenck (1985), el Psicoticismo es el rasgo de personalidad más claramente asociado con la creatividad (Aguilar-Alonso, 1996; Furnham y Nederstrom, 2010; Woody y Claridge, 1977). Este rasgo es característico de las personas creativas y también de aquellas con algún trastorno mental; de hecho, muchos poetas, escritores y artistas considerados como creativos padecieron algún tipo de enfermedad mental, así, encontramos, que se asocia en algunas ocasiones con la esquizofrenia (Lloyd-Evans, Batey y Furnham, 2006; Prentky, 2001; Sass, 2001). En cambio, en otras ocasiones también se ha relacionado la creatividad con rasgos de personalidad como es el caso de la Extraversión (Aguilar-Alonso, 1996).

Desde el Modelo de los Cinco Grandes factores de la personalidad de Costa y McCrae (1992), el utilizado en nuestro estudio, se plantea la existencia de cinco factores en los que se 
agrupan las principales características de la personalidad, estos son: Conciencia/Responsabilidad (habilidad para controlar los impulsos y para organizar y planificar las tareas o actividades a realizar); Apertura a la Experiencia (imaginación, curiosidad, independencia de pensamiento, en definitiva, personas con apertura tanto en las ideas que manifiestan, como en la forma de afrontar los desafíos ante nuevas experiencias o situaciones); Extraversión (sociabilidad, asertividad y eficacia en las interacciones sociales); Amabilidad (sensibilidad ante las necesidades que muestran los demás); e Inestabilidad emocional (tristeza, manifestación de ansiedad e irritabilidad). En este trabajo nos proponemos analizar qué rasgos de la personalidad, valorados con este modelo están más asociados con la creatividad. Para ello, previamente se ha realizado una revisión de la literatura, recogiendo investigaciones con un planteamiento similar, en las que se ha valorado la personalidad con el mismo modelo que el utilizado en nuestro trabajo y estudiando la relación que esos factores tienen con la creatividad, así como las diferencias en personalidad en función del nivel de creatividad que presentan los individuos.

En este sentido, el estudio de King, Walker y Broyles (1996) tuvo como objetivo estudiar la relación entre la creatividad, medida con el TTCT (verbal y figurativo) y la personalidad. Los participantes presentaban edades comprendidas entre los 17 y los 47 años. Sus resultados apuntaron correlaciones significativas entre la creatividad verbal, la Extraversión y la Apertura a la experiencia. Para profundizar en la influencia que la Apertura a la experiencia tiene sobre la creatividad, dividieron la muestra en tres grupos (alta, media y baja creatividad). Los resultados evidenciaron que la habilidad creativa está positivamente relacionada con el logro creativo en niveles medios y altos de Apertura a la experiencia, y que aquellos sujetos que puntúan alto en estas dos variables son los que obtienen los mayores logros creativos. Concretamente, en aquellos individuos con puntuaciones altas en habilidad creativa, y bajas puntuaciones en Apertura a la experiencia, se evidenciaban pocos comportamientos creativos. Afirmando que la Apertura a la experiencia modera la relación entre la habilidad creativa y el logro creativo.

Los resultados de la investigación de Wolfradt y Pretz (2001) también corroboran que es el rasgo de Apertura a la experiencia el que está positivamente relacionado con la creatividad independientemente del instrumento utilizado. Indicando que los rasgos de la personalidad altamente relacionados con la creatividad se constituyen como requisitos necesarios para llegar a destacar dentro de un área de conocimiento. Como ejemplo recogen que, asumiendo que sus participantes más creativos tienen la misma probabilidad de llegar a ser expertos en su área de conocimiento, el poseer rasgos como la Apertura a la experiencia, una baja 
Conciencia/Responsabilidad y un estilo de pensamiento intuitivo, sería considerado como condiciones previas a una personalidad creativa madura. Es decir, es probable que el tener estas precondiciones conduzca a estos individuos a obtener un adecuado nivel de conocimiento experto, a mantenerse motivados para perseguir sus intereses, y si el clima es el apropiado para el individuo, a llegar a tener un alto impacto en su área de conocimiento.

Resultados similares a los anteriores fueron recogidos por Dollinger, Urban y James (2004), siendo la Apertura a la experiencia la variable que correlaciona significativamente con la mayoría de las medidas de creatividad, concretamente con las de creatividad autoinformada.

Szobiová (2006) utilizó una población de 370 adolescentes (196 varones y 174 mujeres) con una edad media de 18 años; y una adaptación del Test de Torrance (TTCT; Torrance, 1974) a población eslovaca (Jurčová, 1984) para valorar la creatividad. Esta autora dividió la muestra en tres grupos (alumnos con baja, media y alta creatividad). Los grupos estaban en función de la puntuación que obtenían en la originalidad del Juego de las Figuras Incompletas del TTCT. Aunque no halló diferencias estadísticamente significativas entre los tres grupos creativos, para los diferentes rasgos de la personalidad. Pero cuando valoró las diferencias en función del sexo, encontró que estas eran significativas en la dimensión de Apertura y Neuroticismo a favor de las chicas. Lo que indicaría, según la autora que, las chicas muestran un mayor interés por las nuevas experiencias e impresiones y una mayor sensibilidad para experimentar emociones agradables y desagradables.

En cuanto a las correlaciones entre la personalidad de los chicos creativos fueron significativas entre los rasgos de Extraversión y Conciencia/Responsabilidad. Mientras que en el caso de las chicas estas fueron significativas entre el Neuroticismo y la Conciencia/Responsabilidad, aunque con una relación negativa (Szobiová, 2006). Todo ello apunta a que son las chicas creativas las que tienen una mayor Conciencia/Responsabilidad, son persistentes, sistemáticas, más estables físicamente, más seguras de sí mismas, y menos ansiosas. Los chicos creativos, sin embargo, son más extravertidos (más activos, sociables y optimistas) y presentan también una mayor Conciencia/Responsabilidad. Dados los resultados obtenidos, la autora afirma que la dimensión Conciencia/Responsabilidad tiene un papel importante en la personalidad creativa. Esta dimensión se encontraría conectada con el Neuroticismo en el caso de las chicas, y con la Extraversión en el caso de los chicos. Cabe destacar que es el grupo de las 
chicas con mayor creatividad el que obtuvo puntuaciones significativamente más altas en el rasgo de Apertura a la experiencia respecto a los chicos más creativos.

También con estudiantes adolescentes, Hoseinifar et al. (2011) analizaron la relación entre la creatividad y los cinco factores de la personalidad en una muestra de 630 estudiantes y entre los resultados encontraron correlaciones significativas de magnitud alta y positivas entre la puntuación total de creatividad y los rasgos de Apertura a la experiencia, Extraversión, Amabilidad y Conciencia/Responsabilidad, mientras que en el caso de Neuroticismo estas correlaciones fueron negativas. A nivel predictivo, obtuvieron que este modelo de personalidad predecía un $48 \%$ de la varianza de la creatividad, entrando los cinco rasgos de personalidad como predictores, la Apertura, Extraversión, Amabilidad y Conciencia/Responsabilidad como predictores positivos y el Neuroticismo como predictor negativo de la misma. Mientras que, Chamorro-Premuzic y Reichenbacher (2008) realizaron un estudio en el que analizaron el efecto predictivo de estos cinco grandes rasgos de la personalidad sobre el pensamiento divergente, en una muestra de 82 estudiantes de psicología. Hallando dos claros predictores como son la Apertura a la experiencia y la Extraversión. Resultados en la línea obtuvieron Sanz de Acedo, Sanz de Acedo y Closas (2014), con una muestra de 180 universitarios y utilizando el CREA (Corbalán et al., 2003) para valorar la creatividad. Obteniendo que la personalidad predice de forma significativa la creatividad ideacional.

En cambio, McCrae e Ingraham (1987) indicaron que la creatividad correlacionaba con la Apertura a la experiencia, pero no con los demás rasgos de la personalidad (Extraversión, Amabilidad, Conciencia/Responsabilidad y Neuroticismo). Resultados similares obtuvieron Furnham, Hughes, y Marshall (2013), quienes confirmaron su hipótesis de estudio relativa a que la Extraversión y la Apertura se mostrarían relacionadas de forma positiva con la creatividad en una muestra de 207 participantes de 16 a 54 años. En esta línea, Kaufman et al. (2016) también confirmaron su hipótesis en cuanto a que la Apertura y la Extraversión predicen el logro creativo en las artes y la inteligencia lo predice en las ciencias en un total de 1035 participantes divididos en 4 muestras, de edades comprendidas entre los 16 y 61 años.

Sin embargo, Krumm, Lemos, y Richaud (2018) analizaron también las relaciones entre la creatividad y la personalidad en una muestra de 359 escolares de 9 a 13 años de edad. La creatividad fue evaluada usando los siguientes instrumentos: la prueba de figuras del Test de Pensamiento Creativo de Torrance, Forma B (TTCT), y la Escala de Personalidad Creadora 
(EPC). Los resultados muestran que únicamente el Neuroticismo se relaciona de forma negativa con la creatividad.

Con población universitaria, Elisondo, Donolo y Corbalán (2009) tuvieron por objetivo analizar las relaciones entre la creatividad y la personalidad en una muestra de 132 alumnos. Partieron de las siguientes hipótesis: a) correlaciones elevadas y significativas entre los rasgos de personalidad (Apertura a la Experiencia y Extraversión) y la creatividad y b) los alumnos potencialmente más creativos logran puntuaciones superiores al resto en Apertura a la experiencia y Extraversión. Los resultados indicaron una sola correlación significativa de signo negativo entre la creatividad y la personalidad medida con el BFQ (Cuestionario Big Five; Caprara, Barbaranelli y Borgogni, 1993), específicamente fue entre el control de impulsos y la Lámina A del CREA (Corbalán et al., 2003). Al analizar las correlaciones entre el CEP (Cuestionario de Personalidad; Pinillos, 1957) observaron correlaciones positivas y estadísticamente significativas entre Extraversión y la Lámina A del CREA. Además, hubo diferencias significativas entre los alumnos potencialmente más creativos y los de creatividad media en la subescala Escrupulosidad (perteneciente a la escala Tesón) y en la escala Tesón del BFQ. Los alumnos de creatividad alta (según Lámina A) obtuvieron puntuaciones significativamente más bajas en dichas escalas. Mientras que con el CEP fueron los estudiantes potencialmente más creativos los que puntuaron más alto en Extraversión que sus compañeros. Cumpliéndose en parte una de las hipótesis de partida (correlaciones significativas y positivas entre creatividad y Extraversión).

Posteriormente, también con el instrumento de creatividad CREA (Corbalán et al., 2003), Limiñana, Corbalán y Sánchez-López (2010) analizaron el comportamiento creativo y su relación con los estilos de personalidad, en una muestra de 80 estudiantes universitarios. Para valorar la personalidad utilizaron el MIPS (Millon, 2001) y para analizar las diferencias dividieron la muestra en tres grupos en función de su nivel de creatividad. Obtuvieron que los estudiantes con mayor creatividad presentan diferencias estadísticamente significativas con respecto a sus compañeros en la dimensión de Extraversión, así como en otras dimensiones motivacionales y de sociabilidad.

Finalmente, Ma (2009) realizó un meta-análisis y observó que la creatividad se encuentra más asociada con la Apertura a la experiencia, pero también, aunque con tamaños del efecto inferiores, con una gran Extraversión, con la Estabilidad emocional, con la 
Conciencia/Responsabilidad y con la Amabilidad; en otras investigaciones como la de Feist (1998) se mantiene que las variables de Conciencia/Responsabilidad y de Amabilidad se encuentran negativamente relacionadas con la creatividad. En este sentido, da Costa et al. (2015) apuntan que una baja puntuación en Neuroticismo, en Amabilidad y en Conciencia/Responsabilidad están globalmente más asociadas con la creatividad. Así como que, la Extraversión es un rasgo asociado moderadamente con la creatividad, apuntando que las personas más enérgicas, es más posible que busquen nuevas formas de resolver los problemas y las tareas, lo cual les conducirá a un mayor logro creativo. Mientras que el rasgo más asociado con la creatividad es la Apertura a la experiencia, debido probablemente a la preferencia por adoptar nuevas perspectivas y ser más flexibles.

De manera que, los estudios analizados indican que el rasgo que caracteriza a las personas más creativas es la Apertura a la experiencia, es decir, las personas abiertas a las nuevas experiencias, y con una mayor independencia de juicio son las que presentan mayores rendimientos creativos (Chamorro-Premuzic, 2006; Dollinger et al., 2004; George y Zhou, 2001; Hoseinifar et al., 2011; King et al., 1996; McCrae y Ingraham, 1987; Wolfradt y Pretz, 2001). Y en menor medida, la Extraversión, es decir aquellas personas que son más sociables, enérgicas y optimistas también son más creativas (Elisondo et al., 2009; Furnham y Nederstrom, 2010; King et al., 1996; Martindale y Dailey, 1996; Sen y Hagtvet, 1993; Stavridou y Furnham, 1996; Wolfradt \& Pretz, 2001). Este dato resulta sorprendente, ya que las creencias en torno a las personas más creativas, es que suelen aislarse, especialmente cuando se encuentran trabajando en una determinada idea. Pero, esta concepción no se encuentra igualmente asociada a todos los ámbitos profesionales, por ejemplo, a los científicos se les percibe como más introvertidos, mientras que los artistas son percibidos como más sociables (Feist, 1998).

Debido a estos resultados que repetidamente han aparecido en la literatura científica, Feist $(2010$; 2019) plantea para analizar la relación entre la personalidad y la creatividad, un modelo con dos grandes factores de personalidad: la Plasticidad y la Estabilidad, que agruparían los Cinco Grandes de personalidad. Es decir, la Plasticidad está compuesta por los rasgos de Apertura y Extraversión, y la Estabilidad, se compone por la Conciencia/Responsabilidad, la Amabilidad y la Inestabilidad emocional. Indicando que es la Plasticidad el factor más relacionado con la creatividad. Así como la capacidad predictiva que la Plasticidad presenta sobre diferentes medidas de creatividad. 


\section{Objetivos e hipótesis}

El objetivo de este trabajo es estudiar qué rasgos de personalidad diferencian a los adolescentes con una alta creatividad de sus compañeros de baja y media creatividad, así como la relación que se establece entre los dos constructos objeto de nuestro estudio.

\section{Método}

\section{Participantes}

En este estudio han participado 217 estudiantes de la ESO de la Región de Murcia (España), quedando una muestra final de 178 estudiantes, al suprimir aquellos casos que tenían datos perdidos en alguno de los instrumentos de medida utilizados, suponiendo esto el $18 \%$ de los participantes. La muestra pertenecía a tres centros educativos, de los cuales un $46 \%$ son de titularidad pública y un 54\% concertada. Las edades estaban comprendidas entre los 12 y los 17 años $(M=14.52 ; D T=1.42)$, de los cuales un $49.3 \%$ son varones. El nivel socioeconómico y cultural de los participantes es medio y el procedimiento de muestreo utilizado ha sido de tipo incidental. La distribución de estos alumnos en función del curso académico queda recogida en la Tabla 1.

Tabla 1. Distribución de los estudiantes según el curso académico

\begin{tabular}{lcccc}
\hline & $1^{\circ}$ de la ESO & $2^{\circ}$ de la ESO & $3^{\circ}$ de la ESO & $4^{\circ}$ de la ESO \\
\hline Frecuencia & 24 & 11 & 76 & 67 \\
Porcentaje & $13.5 \%$ & $6.2 \%$ & $42.7 \%$ & $37.6 \%$ \\
\hline
\end{tabular}

\section{Instrumentos}

1. Para valorar la personalidad se han utilizado dos instrumentos de medida, debido a la amplitud en cuanto a edad cronológica de los participantes. Para aquellos estudiantes que tenían entre 12 y 15 años se utilizó el BFQ-NA (Barbaranelli et al., 1998) y para los que su edad estaba comprendida entre los 16 y 17 años se les administró el NEO-FFI (Costa \& McCrae, 2008). Se seleccionaron ambos instrumentos por estar basados en el Modelo de los Cinco Grandes.

a) Big Five Questionnaire de Personalidad para niños y adolescentes (BFQ-NA; Barbaranelli et al., 1998); en concreto, la adaptación al español (Del Barrio et al., 2006). Es una prueba de evaluación destinada a la medición de la personalidad infantil y adolescente (8-15 años). Está compuesta por 65 ítems a los que se responde a través de una escala tipo Likert con 
cinco alternativas que van desde casi siempre a casi nunca. Estos ítems se clasifican en las cinco dimensiones descritas en el modelo de Los Cinco Grandes que son: Conciencia/Responsabilidad, Amabilidad, Extraversión, Inestabilidad emocional y Apertura a la experiencia. La fiabilidad obtenida por Del Barrio et al. (2006) es adecuada con puntuaciones que oscilan entre .78 y .88 .

b) Inventario de Personalidad NEO Revisado, versión reducida (NEO-FFI; Costa \& McCrae, 2008). Está compuesto por 60 ítems con cinco opciones de respuesta, que van desde en total desacuerdo a totalmente de acuerdo. La edad de aplicación es a partir de los 16 años. Este instrumento evalúa cinco factores de personalidad: Responsabilidad o Tesón, Amabilidad o Afabilidad, Extraversión, Neuroticismo versus Estabilidad emocional, y Apertura a la experiencia. En cuanto a la fiabilidad de la prueba los autores obtuvieron coeficientes que varían entre .63 y .79 .

2. Para evaluar la creatividad se ha utilizado el Test de Pensamiento Creativo de Torrance (TTCT; Torrance, 1974). Evalúa el nivel de creatividad de los alumnos a través de dos sub-pruebas, una verbal y otra figurativa. En este estudio hemos utilizado la figurativa. En ambas formas se evalúa la fluidez (número de respuestas que dan los alumnos), flexibilidad (variedad de las respuestas), originalidad (respuestas que son novedosas) y elaboración (detalles que se proporcionan para embellecer el producto creativo). De los tres juegos que componen la prueba de creatividad figurativa, en el presente estudio hemos utilizado el Juego 3: "Líneas Paralelas". Se ha seleccionado este Juego, debido a que es el que mejor evalúa las cuatro habilidades de la creatividad (Ferrando, Ferrándiz, Bermejo, Sánchez, Parra \& Prieto, 2007; Oliveira, Almeida, Ferrándiz, Ferrando, Sainz \& Prieto, 2009). La fiabilidad de la prueba obtenida para este estudio ha sido un coeficiente alfa de Cronbach de .782 y a través del coeficiente de dos mitades de Guttman fue de .787 .

\section{Procedimiento}

En primer lugar, se seleccionaron los centros educativos, así como, los cursos que iban a ser evaluados. En segundo lugar, se contactó con los centros, se elaboró un calendario de aplicación de pruebas, atendiendo a la disponibilidad horaria de los centros, además de una autorización dirigida a los padres de los alumnos que iban a participar en el estudio, con la finalidad de informar sobre el objetivo del estudio y obtener la autorización. En tercer lugar, se aplicaron las diferentes pruebas en horario lectivo por evaluadores con experiencia en las áreas 
de Psicología y Pedagogía. En cuarto lugar, se corrigieron las pruebas administradas y se informatizaron las respuestas, y finalmente, se procedió a realizar los análisis estadísticos y extraer las conclusiones del estudio.

\section{Análisis de datos}

El diseño utilizado ha sido de tipo cuantitativo no experimental. Los análisis estadísticos llevados a cabo para acometer los objetivos del presente estudio fueron: análisis de fiabilidad del instrumento de creatividad utilizado (el juego 3 del TTCT), análisis descriptivos, análisis mediante el coeficiente de correlación de Pearson, para estudiar la relación entre las dimensiones utilizadas en este estudio, y análisis de comparación de medias (ANOVA de un factor), entre los diferentes grupos creativos, todos ellos realizados a través del programa estadístico SPSS versión 24 para Windows.

\section{Resultados}

En primer lugar, se analizaron los estadísticos descriptivos de las variables del estudio, así como las puntuaciones mínimas y máximas, y los valores de asimetría y curtosis (ver Tabla 2).

Tabla 2. Estadísticos descriptivos de las variables del estudio

\begin{tabular}{|c|c|c|c|c|c|c|}
\hline & Mínimo & Máximo & Media & $D T$ & Asimetría & Curtosis \\
\hline $\begin{array}{l}\text { Conciencia/Respon- } \\
\text { sabilidad/Responsa- } \\
\text { bilidad }\end{array}$ & 6.00 & 92.00 & 52.75 & 21.61 & -.203 & -1.285 \\
\hline Apertura & 11.00 & 39.00 & 26.20 & 5.10 & -.213 & -.033 \\
\hline Extraversión & 18.00 & 50.00 & 37.63 & 6.70 & -.540 & -.077 \\
\hline Amabilidad & 14.00 & 50.00 & 34.84 & 7.14 & -.297 & -.326 \\
\hline $\begin{array}{l}\text { Inestabilidad emo- } \\
\text { cional/Neuroticismo }\end{array}$ & 6.00 & 47.00 & 25.16 & 8.33 & .283 & -.208 \\
\hline Fluidez TTCT & 1.0 & 30.0 & 16.35 & 7.21 & .247 & -.764 \\
\hline Flexibilidad TTCT & 3.0 & 23.0 & 11.79 & 4.38 & .039 & -.651 \\
\hline Originalidad TTCT & 3.0 & 66.0 & 29.54 & 14.68 & .288 & -.631 \\
\hline Elaboración TTCT & 1.0 & 67.0 & 23.94 & 12.63 & .706 & .690 \\
\hline
\end{tabular}


Analizadas las puntuaciones medias obtenidas por los estudiantes, cabe destacar que la media del rasgo de Conciencia/Responsabilidad es la más elevada, mientras que las puntuaciones en los otros cuatro rasgos de la personalidad obtienen medias muy similares. Mientras que si nos centramos en las puntuaciones de creatividad, observamos que las medias más elevadas son las de originalidad y elaboración. Si atendemos a las puntuaciones mínimas y máximas y a la distribución de las puntuaciones, se observó que siguen una distribución normal. Respecto a las puntuaciones de asimetría y curtosis, cabe indicar que se encuentran en los límites adecuados, tal como establece la literatura científica, a excepción de la puntuación de curtosis del rasgo de Conciencia/Responsabilidad, que apunta a la presencia de puntuaciones extremas en esta variable.

Al objeto de profundizar en la relación que se establece entre los constructos de nuestro estudio, realizamos un análisis de correlación de Pearson, recogido en la Tabla 3.

Tabla 3. Correlación entre las variables del estudio

\begin{tabular}{lcccc}
\hline & $\begin{array}{c}\text { Fluidez } \\
\text { TTCT }\end{array}$ & $\begin{array}{c}\text { Flexibilidad } \\
\text { TTCT }\end{array}$ & $\begin{array}{c}\text { Originalidad } \\
\text { TTCT }\end{array}$ & $\begin{array}{c}\text { Elaboración } \\
\text { TTCT }\end{array}$ \\
\hline $\begin{array}{l}\text { Conciencia/Responsabili- } \\
\text { dad/Responsabilidad }\end{array}$ & $.163^{*}$ & .077 & .142 & -.091 \\
Apertura & .106 & .059 & .096 & $.161^{*}$ \\
Extraversión & $.210^{* *}$ & $.160^{*}$ & $.180^{*}$ & .033 \\
Amabilidad & .097 & .042 & .086 & -.047 \\
$\begin{array}{l}\text { Inestabilidad emocional/Neuro- } \\
\text { ticismo }\end{array}$ & .037 & .019 & .002 & -.064 \\
\hline
\end{tabular}

**. La correlación es significativa al nivel .01 (bilateral).

*. La correlación es significante al nivel .05 (bilateral).

$\mathrm{Al}$ observar los resultados cabe destacar que aunque hay relaciones estadísticamente significativas entre los rasgos de personalidad y las variables de creatividad, estas son de magnitud baja o moderada. En este sentido, el rasgo de la personalidad que más se relaciona con las variables creativas es la Extraversión, ya que presenta relaciones estadísticamente significativas y de signo positivo con tres de las cuatro dimensiones contempladas en el estudio de la creatividad, esto es, con las puntuaciones de Fluidez, Flexibilidad y Originalidad. Otros rasgos de la personalidad que también se relacionan con algunas de las dimensiones de la creatividad, es el 
caso de la Conciencia/Responsabilidad con la Fluidez de ideas, y de la Apertura a la experiencia con la Elaboración. Destacar en este sentido, que la relación más significativa y de magnitud más elevada como se observa en la Tabla 3 se da entre el rasgo de Extraversión y la dimensión de Fluidez de ideas.

Estas relaciones nos indican que ambas características personales (personalidad y creatividad) presentan ciertas asociaciones entre ellas. Por ello, nos planteamos si aquellas personas que presentan un mayor potencial creativo tienen también un perfil de personalidad diferente de aquellos que presentan un menor nivel de creatividad.

En la Tabla 4, podemos observar los estadísticos descriptivos (medias y desviaciones típicas) de las puntuaciones obtenidas por los participantes de la muestra en función del nivel de creatividad. Para ello, se dividió la muestra en tres grupos en función de las puntuaciones de creatividad (alta, media y baja creatividad) obtenidas en el juego 3 "Líneas paralelas" del TTCT (Torrance, 1974). Y se realizó la prueba de Levene para comprobar la homogeneidad de las varianzas de las variables utilizadas, hallando que únicamente es la Extraversión la que no cumple este supuesto.

Tabla 4. Estadísticos descriptivos de la personalidad según los niveles de creatividad

\begin{tabular}{lccccc}
\hline & $\begin{array}{c}1 \\
(\mathrm{~N}=45)\end{array}$ & $\begin{array}{c}2 \\
(\mathrm{~N}=91)\end{array}$ & $\begin{array}{c}3 \\
(\mathrm{~N}=42)\end{array}$ & & \\
& $\begin{array}{c}\text { Media } \\
(D T)\end{array}$ & $\begin{array}{c}\text { Media } \\
(D T)\end{array}$ & $\begin{array}{c}\text { Media } \\
(D T)\end{array}$ & Anova & Hoc \\
\hline Conciencia/Respon- & & & & & \\
sabilidad/ Responsa- & 52.33 & 50.42 & 61.86 & $\mathrm{~F}(2,175)=4.400 ; p=.014$ & $3>2$ \\
bilidad & $(19.88)$ & $(21.05)$ & $(21.77)$ & & \\
& & & & & \\
Apertura & 26.07 & 25.65 & 27.48 & $\mathrm{~F}(2,175)=1.848 ; p=.161$ & \\
& $(5.05)$ & $(5.48)$ & $(4.31)$ & & $3>2$ \\
Extraversión* & 36.22 & 37.34 & 40.19 & $\mathrm{~F}(2,175)=4.309 ; p=.015$ & $3>2$ \\
& $(7.21)$ & $(6.86)$ & $(4.92)$ & & \\
Amabilidad & 35.73 & 33.35 & 37.86 & $\mathrm{~F}(2,175)=6.404 ; p=.002$ & $3>19$ \\
Inestabilidad emo- & $(6.65)$ & $(6.90)$ & $(7.25)$ & & \\
cional/ & 24.31 & 26.19 & 24.62 & $\mathrm{~F}(2,175)=.937 ; p=.394$ & \\
Neuroticismo & $(8.11)$ & $(8.81)$ & $(8.12)$ & & \\
\hline
\end{tabular}

Nota: 1: Grupo de Baja Creatividad; 2: Grupo de Creatividad Media; 3: Grupo de Alta Creatividad *No se asume igualdad de varianzas 
A nivel descriptivo podemos observar que son los alumnos con más creatividad los que obtienen puntuaciones más elevadas en cuatro de los cinco rasgos de la personalidad valorados (Conciencia/Responsabilidad, Apertura, Extraversión y Amabilidad). En cambio, en el rasgo de Inestabilidad emocional son los estudiantes con creatividad media los que obtienen las medias más elevadas.

Pero para saber si estas diferencias observadas son estadísticamente significativas o no, pasamos a realizar un Anova de un factor. En la Tabla 4, también podemos observar que las diferencias estadísticamente significativas en personalidad según el nivel de creatividad se dan en Conciencia/Responsabilidad, Extraversión y Amabilidad. Para conocer entre qué grupos creativos (alta, media o baja creatividad) se dan estas, realizamos los análisis Post Hoc (DMS en las variables en las que se asumen igualdad de varianzas, y T3 Dunnett, en las que no se asume) que indican que estas diferencias se dan en el caso de la Conciencia/Responsabilidad y de la Amabilidad entre los estudiantes más creativos y sus compañeros con una creatividad media, a favor de los primeros. Y en el caso de la Extraversión, se dan a favor de los estudiantes más creativos con respecto a sus compañeros con media y baja creatividad.

\section{Discusión y Conclusiones}

Los resultados obtenidos en este trabajo apuntan, por una parte, que el rasgo más relacionado con la creatividad es la Extraversión y en menor medida la Conciencia/Responsabilidad y la Apertura. Por otra, cabe destacar que son los rasgos de Conciencia/Responsabilidad, Amabilidad y Extraversión los que más diferencian a los alumnos del grupo con mayor creatividad con respecto a sus compañeros de media y baja creatividad. Estos resultados discrepan con los obtenidos por King et al. (1996), que hallaron diferencias entre los grupos creativos sólo en la variable Apertura. Y con Szobiová (2006), quien no halló diferencias entre los grupos creativos en ningún rasgo de la personalidad.

Como hemos recogido en la revisión de la literatura, otros trabajos apuntan resultados muy diferentes a los de este estudio al encontrar relaciones principalmente entre la creatividad y variables de personalidad como la Apertura a la experiencia (Chamorro-Premuzic, 2006; da Costa et al., 2015; Dollinger et al., 2004; George y Zhou, 2001; King et al., 1996; Wolfradt y Pretz, 2001). Otros trabajos también mostraron que no había un único rasgo que se relacionara en mayor medida con la creatividad, sino que era la combinación de algunos de ellos, lo que 
definía mejor el comportamiento o rendimiento creativo, este es el caso de la Extraversión y la Apertura para Chamorro-Premuzic y Reichenbacher (2008). En esta línea, Feist (2019) plantea que la Plasticidad, entendida como la combinación de Apertura a la experiencia y Extraversión, se relaciona y predice en mayor medida el pensamiento y la conducta creativa.

Podemos concluir, que según nuestros resultados las variables de Extraversión y Conciencia/Responsabilidad se constituyen como rasgos fundamentales para la identificación del perfil creativo. En el caso de nuestros estudiantes, el tener una alta puntuación en Conciencia/Responsabilidad no inhibe su manifestación creativa, sino que por el contrario, es un facilitador del rendimiento creativo. Estos resultados están en la línea de los hallados por autores como Furnham (2015), Ma (2009) y Szobiová (2006) quienes obtuvieron relaciones positivas entre los rasgos de Extraversión y Conciencia/Responsabilidad y la Creatividad.

También, en el caso de la Extraversión, estudios como los de Dollinger et al. (2004), King et al. (1996), y Martindale y Dailey (1996), hallaron relaciones positivas y significativas entre este rasgo de la personalidad y la Creatividad. Además, Furnham y Bachtiar (2008), utilizando cuatro medidas de creatividad, hallaron una correlación significativa entre la Extraversión y la creatividad. Estos resultados están en la línea de los que hemos obtenido en este trabajo, utilizando el TTCT (Torrance, 1974) para valorar la creatividad.

En el caso de la Conciencia/Responsabilidad son numerosos los estudios que encuentran correlaciones con la creatividad, pero de signo negativo. Este es el caso de estudios como el de Feist (1998), da Costa et al. (2015), y George y Zhou (2001). Resultados que difieren con los encontrados en nuestro estudio, ya que la correlación hallada es positiva.

Un resultado llamativo de nuestro estudio es el obtenido en cuanto a la Amabilidad. Esta es una variable que en la mayoría de estudios no suele aparecer relacionada con el perfil creativo. A excepción del trabajo realizado por Ma (2009), en el que indicó relación entre todos los rasgos de la personalidad y la creatividad. La Amabilidad es un rasgo de la personalidad muy ligado a la tolerancia y a tener una predisposición a establecer buenas interacciones sociales.

Algunas de las limitaciones de este trabajo es el reducido tamaño muestral y la utilización de un solo instrumento para evaluar el potencial creativo de los estudiantes. Aunque el TTCT es un instrumento ampliamente utilizado y con adecuadas propiedades psicométricas (Ferrando 
et al., 2007; Kim, Cramond y Bandalos, 2006; Oliveira et al., 2009). Incluso los resultados del trabajo de Prieto, López, Ferrándiz y Bermejo (2003) también indican que es una medida adecuada para la valoración de la creatividad en los primeros niveles instruccionales (Educación Infantil y $1^{\circ}$ de Educación Primaria).

En futuros estudios sería interesante analizar la capacidad predictiva de la personalidad sobre la creatividad a través de un estudio longitudinal. Ya que, como indican diferentes autores como Sternberg y Lubart (1995), Csikszentmihalyi (1990) o Amabile (1983), el contexto influye en el desarrollo y manifestación de la creatividad, y para poder alcanzar logros creativos en diferentes dominios de conocimiento o en distintas actividades de la vida cotidiana (Ivcevic, 2007) son necesarios factores como el entrenamiento, favorecido por el contexto, la motivación intrínseca y un alto potencial creativo (Feist, 2013).

\section{Agradecimientos}

Este trabajo se ha realizado con la ayuda de la Subdirección General de Proyectos de Investigación I+D+i. Ministerio de Ciencia y Tecnología (España). (Ref: EDU2014-53646-R).

\section{Referencias}

Aguilar-Alonso, A. (1996). Personality and creativity. Personality and Individual Differences, 21(6), 959969.

Amabile, T. M. (1983). The social psychology of creativity. New York: Springer-Verlag.

Amabile, T. M., Barsade, S. G., Mueller, J. S., \& Staw, B. M. (2005). Affect and creativity at work. Administrative science quarterly, 50(3), 367-403. https://doi.org/10.2189/asqu.2005.50.3.367

Barbaranelli, C., Caprara, G.V. \& Rabasca, A. (1998). Manuale del BFQC. Big Five Questionnaire Children. O.S. Organizzaaioni Speciali-Firenze.

Bernal, A., Esparza, J., Ruiz, M.J., Ferrando, M., \& Sainz, M. (2017). Especificidad de la Creatividad: Figurativa y Científica. Electronic Journal of Research in Educational Psychology, 15(3), 574-597. http://dx.doi.org/10.14204/ejrep.43.16094.

Caprara, G., Barbaranelli, J. y Borgogni, L. (1993). BFQ: cuestionario "Big Five”. $2^{\text {a }}$ Edición revisada y ampliada en español (1998). TEA Ediciones. Madrid.

Chamorro-Premuzic, T. (2006). Creativity versus conscientiousness: Which is a better predictor of student performance? Applied Cognitive Psychology, 20(4), 521-531. https://doi.org/10.1002/acp.1196 
Chamorro-Premuzic, T. \& Reichenbacher, L. (2008). Effects of personality and threat of evaluation on divergent and convergent thinking. Journal of Research in Personality, 42(4), 1095-1101. https://doi.org/10.1016/j.jrp.2007.12.007

Corbalán Berná, J., F. Martínez Zaragoza, D. S. Donolo, C. Alonso Monreal, M., Tejerina Arreal y M. R. Limiñana Gras (2003). CREA. Inteligencia Creativa. Una medida Cognitiva de la Creatividad. TEA Ediciones: Madrid.

Costa, P. T., \& McCrae, R. R. (1992). Revised NEO personality Inventory: Professional manual. Odessa, FL: Psychological Assessment Resources.

Costa, P. T., \& McCrae, R. R. (2008). NEO-PI-R, Inventario de Personalidad NEO Revisado y NEO-FFI, Inventario NEO Reducido de Cinco Factores. Madrid: TEA Ediciones, S.A.

Csikszentmihalyi, M. (1990). The domain of creativity. In M. A. Runco \& R. S. Albert (Eds.). Theories of Creativity (pp. 190-212). Newbury Park, C. A: Sage.

da Costa, S., Páez, D., Sánchez, F., Garaigordobil, M., \& Gondim, S. (2015). Personal factors of creativity: A second order meta-analysis. Journal of Work and Organizational Psychology, 31, 165-173. https://doi.org/10.1016/j.rpto.2015.06.002

Del Barrio, V., Carrasco, M. A., \& Holgado, F. P. (2006). BFQ-NA. Cuestionario "Big Five" de personalidad para niños y adolescentes. Madrid: TEA Ediciones, S. A.

Dollinger, S. J., Urban, K. K., \& James, T. A. (2004). Creativity and openness: further validation of two creative product measures. Creativity Research Journal, 16(1), 35-47. https://doi.org/10.1207/s15326934crj1601_4

Elisondo, R. C., Donolo, D. S., \& Corbalán, F. J. (2009). Evaluación de la Creatividad ¿Relaciones con inteligencia y personalidad?. Revista Iberoamericana de Diagnóstico y Evaluación e Avaliação Psicológica, 2(28), 67-79.

Eysenck, H. J., \& Eysenck, M. W. (1985). Personality and individual differences: A natural science approach. New York: Plenum. https://doi.org/10.1007/978-1-4613-2413-3

Feist, G. J. (1998). A meta-analysis of personality in scientific and artistic creativity. Personality and Social Psychology Review, 2(4), 290-309. https://doi.org/10.1207/s15327957pspr0204_5

Feist, G. J. (2010). The function of personality in creativity. In J. C. Kaufman \& R. J. Sternberg (Eds.), The Cambridge handbook of creativity (pp. 113-130). New York: Cambridge University Press. https://doi.org/10.1017/cbo9780511763205.009

Feist, G. J. (2013). The Scientific Personality. In G.J., Feist, \& M.E. Gorman (Eds.), Handbook of the Psychology of Science (pp. 95-121). New York: Springer Publishing Company.

Feist, G. J. (2018). In search of the Creative Personality. En R. J. Sternberg y J. C. Kaufman (Eds.), The nature of human creativity (63-76). United Kingdom: Cambridge University Press. https://doi.org/10.1017/9781108185936.007

Feist, G. J. (2019). Creativity and the Big Two model of personality: plasticity and stability. Current Opinion in Behavioral Sciences, 27, 31-35. https://doi.org/10.1016/j.cobeha.2018.07.005 
Feist, G. J., \& Barron, F. X. (2003). Predicting creativity from early to late adulthood: Intellect, potential, and personality. Journal of research in personality, 37(2), 62-88. https://doi.org/10.1016/s0092$\underline{6566(02) 00536-6}$

Ferrando, M., Ferrándiz, C., Bermejo, M. R., Sánchez, C., Parra, J., \& Prieto, M. D. (2007). Estructura interna y baremación del Test de Pensamiento Creativo de Torrance. Psicothema, 19(3), 489-496.

Furnham, A. (2015). The Bright and dark side correlates of creativity: Demographic, ability, personality traits and personality disorders associated with divergent thinking. Creativity Research Journal, 27(1), 3946. https://doi.org/10.1080/10400419.2015.992676

Furnham, A., \& Bachtiar, V. (2008). Personality and intelligence as predictors of creativity. Personality and Individual Differences, 45(7), 613-617. https://doi.org/10.1016/j.paid.2008.06.023

Furnham, A, \& Nederstrom, M. (2010). Ability, demographic and personality predictors of creativity. Personality and Individual Differences, 48(8), 957-961. https://doi.org/10.1016/j.paid.2010.02.030

Furnham, A., Hughes, D. J., \& Marshall, E. (2013). Creativity, OCD, narcissism and the Big Five. Thinking Skills and Creativity, 10, 91-98. https://doi.org/10.1016/j.tsc.2013.05.003

George, J. M., \& Zhou, J. (2001). When openness to experience and conscientiousness are related to creative behavior: an interactional approach. Journal of applied psychology, 86(3), 513-524. https://doi.org/10.1037//0021-9010.86.3.513

Hoseinifar, J., Siedkalan, M. M., Zirak, S. R., Nowrozi, M., Shaker, A., Meamar, E., \& Ghaderi, E. (2011). An investigation of the relation between creativity and five factors of personality in students. Procedia-Social and Behavioral Sciences, 30, 2037-2041. https://doi.org/10.1016/j.sbspro.2011.10.394

Hughes, D. J., Furnham, A., \& Batey, M. (2013). The structure and personality predictors of self-rated creativity. Thinking Skill and Creativity, 9, 76-84. https://doi.org/10.1016/j.tsc.2012.10.001

Ivcevic, Z. (2007). Artistic and everyday creativity: An act-frequency approach. The Journal of Creative Behavior, 41(4), 271-290. https://doi.org/10.1002/j.2162-6057.2007.tb01074.x

Jurčová, M. (1984). Torranceho figurálny test tvorivého myslenia. Bratislava: Psychodiagnostické a didaktické testy.

Kaufman, S. B., Quilty, L. C., Grazioplene, R. G., Hirsh, J. B., Gray, J. R., Peterson, J. B., \& DeYoung, C. G. (2016). Openness to experience and intellect differentially predict creative achievement in the arts and sciences. Journal of personality, 84(2), 248-258. https://doi.org/10.1111/jopy.12156

Kim, K. H., Cramond, B., \& Bandalos, D. L. (2006). The latent structure and measurement invariance of scores on the Torrance Tests of Creative Thinking-Figural. Educational and Psychological Measurement, 66(3), 459-477. doi: https://doi.org/10.1177/0013164405282456

King, L. A., Walker, L. M., \& Broyles, S. J. (1996). Creativity and the five-factor model. Journal of research in personality, 30(2), 189-203. https://doi.org/10.1006/jrpe.1996.0013

Krumm, G., Lemos, V., \& Richaud, M. C. (2018). Personality and creativity: A study in Spanish-speaking children. International Journal of Psychological Research, 11(1), 33-41. https://doi.org/10.21500/20112084.2867 
Limiñana, R. M., Corbalán, J., \& Sánchez-López, M. P. (2010). Creativity and Personality Styles: An approach to creative profiles in university students. Anales De Psicología / Annals of Psychology, 26(2), 273-278.

Lloyd-Evans, R., Batey, M., \& Furnham, A. (2006). Bipolar disorder and creativity: Investigating a possible link. In A. Columbus (Ed.), Advances in psychology research (Vol. 40) (pp. 111-142). New York: Nova Science Publishers, Inc.

Ma, H. (2009). The effect size of variables associated with creativity: A Meta-Analysis. Creativity Research Journal, 21(1), 30-42. https://doi.org/10.1080/10400410802633400

Martindale, C., \& Dailey, A. (1996). Creativity, primary process cognition and personality. Personality and individual differences, 20(4), 409-414. https://doi.org/10.1016/0191-8869(95)00202-2

McCrae, R. R. \& Greenberg, D. M. (2014). Openness to experience. In D. K. Simonton, The Wiley Handbook of Genius (pp. 222-243). UK: Wiley Blackwell.

McCrae, R. \& Ingraham, L. J. (1987). Creativity, Divergent Thinking, and Openness to Experience. Journal of Personality and Social Psychology, 52(6), 1258-1265. 10.1037//0022-3514.52.6.1258

Millon, T. (2001). Inventario de Estilos de Personalidad de Millon, Manual. [Millon Index of Personality Styles, Manual]. Adaptación de M.P. Sánchez-López, J.F. Díaz-Morales y M.E. Aparicio-García. Madrid: TEA Ediciones.

Oliveira, E., Almeida, L., Ferrándiz, C., Ferrando, M., Sainz, M., \& Prieto, M. D. (2009). Tests de pensamiento creativo de Torrance (TTCT): elementos para la validez de constructo en adolescentes portugueses. Psicothema, 21(4), 562-567.

Overskeid, G., Grønnerød, C., \& Simonton, D. K. (2012). The personality of a nonperson: Gauging the inner Skinner. Perspectives on Psychological Science, 7(2), 187-197.

Prieto, M. D., López, O., Ferrándiz, C. y Bermejo, R. (2003). Adaptación de la prueba figurativa del test de pensamiento creativo de Torrance en una muestra de alumnos de los primeros niveles educativos. Revista de Investigación Educativa, 21(1), 201-213.

Pinillos, J. (1957). Cuestionario de Personalidad CEP. Madrid. TEA Ediciones.

Prentky, R. A. (2001). Mental illness and roots of genius. Creativity Research Journal, 13(1), 95-104. https://doi.org/10.1207/s15326934cri1301_11

Romo-Santos, M., Sánchez-Ruiz, M. J., \& Alfonso-Benlliure, V. (2017). Creatividad y personalidad a través de dominios: una revisión crítica. Anuario de Psicología, 47(2), 57-69. https://doi.org/10.1016/j.anpsic.2017.04.003

Sanz de Acedo, M. L., Sanz de Acedo, M. T., \& Closas, A. H. (2014). An explanatory model regarding the relationships between psychological traits and creativity. Anales de Psicología, 30(1), 355-363. http://dx.doi.org/10.6018/analesps

Sass, L. A. (2001). Schizophrenia, modernism, and the "creative imagination": On creativity and psychopathology. Creativity Research Journal, 13(1), 55-74. https://doi.org/10.1207/s15326934crj1301_7 
Sen, A. K., \& Hagtvet, K. A. (1993). Correlations among creativity, intelligence, personality, and academic achievement. Perceptual and motor skills, 77(2), 497-498. https://doi.org/10.2466/pms.1993.77.2.497

Selby, E. C., Shaw, E. J., \& Houtz, J. C. (2005). The creative personality. Gifted Child Quarterly, 49(4), 300314. https://doi.org/10.1177/001698620504900404

Simonton, D. K. (2000). Creativity: Cognitive, developmental, personal, and social aspects. American Psychologist, 55, 151-158. https://doi.org/10.1037//0003-066x.55.1.151

Stavridou, A., \& Furnham, A. (1996). The relationship between psychoticism, trait-creativity and the attentional mechanism of cognitive inhibition. Personality and Individual Differences, 21(1), 143-153. https://doi.org/10.1016/0191-8869(96)00030-x

Sternberg, R. J. \& Lubart, T. (1995/1997). Defying the Crowd: Cultivating Creativity in a Culture of Conformity. Free Press. (Trad. Castellano, La creatividad en una sociedad conformista. Madrid: Paidós).

Szobiová, E. (2006). The five personality dimensions in relation to creative thinking of adolescents. Studia Psychologica, 48, 241-249.

Torrance, E. P. (1974). The Torrance Tests of Creative Thinking: Technical-norm Manual. Scholastic Testing Service: Bensenville, IL.

Wolfradt, U., \& Pretz, J. E. (2001). Individual differences in creativity: Personality, story writing, and hobbies. European Journal of Personality, 15(4), 297-310. https://doi.org/10.1002/per.409

Woody, E., \& Claridge, G. (1977). Psychoticism and thinking. British Journal of Social and Clinical Psychology, 16(3), 241-248. https://doi.org/10.1111/j.2044-8260.1977.tb00225.x

Recibido: 13-09-2019

Aceptado: 13-12-2019 